\title{
DEFLECTION IN ONE SIDE RECORDABLE MAGNETO-OPTICAL DISKS WITH POLYCARBONATE SUBSTRATES INDUCED BY HUMIDITY CHANGES
}

\author{
F. KUROSAWA and S. KATSUDA
}

R\&D Lab., NEC Home Electronics, Ltd., 262 Hisamoto, Takatsu-ku, Kawasaki 213, Japan

\begin{abstract}
One side recordable magneto-optical disks, using PC substrates, deflect due to humidity changes. Such deflection in the magneto-optical disks induces problems in regard to their durability and reliability. The authors developed a new deflection measuring method utilizing strain gages, which can measure precise deflection behaviors for the disks after humidity changes. Deflection behavior was analyzed by solving the diffusion equation of moisture into PC substrates. The experiments and the analysis revealed the diffusion coefficient of moisture in PC substrates and their activation energy.
\end{abstract}

KEY WORDS : ONE SIDE RECORDABLE MAGNETO-OPTICAL DISK, MOISTURE DIFFUSION, DEFLECTION, STRAIN GAGES

\section{INTRODUCTION}

Polycarbonate ( PC ) substrates have better mass productivity and shock resistance than glass substrates. However, PC is so hygroscopic that one side recordable magneto-optical disks using PC substrates, deflect due to humidity changes [1] ( Fig. 1 ). Such deflections in the magneto-optical disks induce problems in regard to their durability and reliability. Therefore, deflection measurement on magneto-optical disks after humidity changes is very important to assure of their durability and reliability. The authors developed a new deflection measuring method utilizing strain gages, which can measure precise deflection behaviors for the disks after humidity changes.

\section{EXPERIMENTAL}

Test disks were prepared by $\mathrm{TbFeCo}$ alloy sputtering and $\mathrm{Si}-\mathrm{N}_{2}$ reactive sputtering on $\mathrm{PC}$ substrates ( $120 \mathrm{~mm}$ in diameter ). Strain gages were attached to both sides of the disks, opposite to each other. Each strain gage was coated with a moisture proofing wax. (Ef-Er)/ 2 and $(E f+E r) / 2$ express deflection strain and stretching strain, respectively, where Ef and Er denote strain gage outputs from the front surface ( light beam incident side ) and rear surface ( sputtered material side), respectively. All measurements were carried out in a programmable thermo-hygrostat chamber or a vacuum chamber. Most measurements were carried out with stepwise humidity changes, for example from $50 \% \mathrm{RH}$ to $90 \%$ RH or vice versa. Before measurements, test disks were left in the thermo-hygrostat chamber in order to saturate the moisture distributions in the disks.

\section{MODELING AND ANALYSIS}

One side recordable magneto-optical disks absorb moisture from only their front surface. The absorption processes were analyzed by solving the diffusion equation of moisture into PC substrates, assuming a diffusion coefficient. Then, the appropriate diffusion coefficients were decided as the ones which best fit the experiments. Figure 2 shows calculated moisture concentration distributions in the PC substrate, induced by a humidity change. Asymmetrical moisture concentration distributions induce the disk deflections. Figure 3 shows deflection and stretching behaviors, calculated from moisture distribution data in Fig. 2, where deflection and stretching strains were assumed to be proportional to moisture concentrations.

\section{RESULTS AND DISCUSSION}

Figure 4 shows typical experimental strain behaviors for the disk induced by humidity change. In this experiment, temperature was kept at $30^{\circ} \mathrm{C}$, whereas relative humidity was changed from $50 \%$ to $90 \%$ ( humidifying ) at time zero. After the humidity change, the disk started deflecting to convexity on the front surface immediately. The deflecting strain had a peak about 4.5 hours after the humidity change. Then, the deflecting strain decreased gradually. Stretching strain (expansion) of the disk increased into saturation. When humidity changed to the opposite ( drying ), the disk started contracting and deflecting to concavity on 
the front surface.

Figure 5 shows deflection strains for a disk induced by evacuation and ventilation. Evacuation induced the same behavior as in the drying process, whereas ventilation induced the same one as in the humidifying process.

Figures 6 and 7 show deflection and stretching behaviors, respectively, in comparison between experiments and analysis. These experiments shows excellent agreement with the analysis.

Figure 8 shows deflection behaviors at $20^{\circ} \mathrm{C}$ $60^{\circ} \mathrm{C}$, where experiments also shows excellent agreement with the analysis. Higher temperature induces faster moisture diffusion. Therefore, higher temperature induces earlier deflection peaks. Moisture diffusion coefficients at each temperature were calculated by comparing deflection behavior curves, between the experimental and analytical values. Figure 9 shows temperature dependence for diffusion coefficients. This Arrhenius plot gives a good linearity, whose apparent activation energy was $41 \mathrm{~kJ} / \mathrm{mol}(0.42 \mathrm{eV})$.

\section{CONCLUSION}

Deflection in one side recordable magnetooptical disks with PC substrates, induced by humidity changes, has been evaluated by utilizing a newly developed measurement method.

Deflection behavior was analyzed by solving the diffusion equation of moisture into $\mathrm{PC}$ substrate. The experiments and analysis showed excellent agreement with each other. Moisture diffusion coefficient in PC substrates and their activation energy were deduced.

\section{REFERENCE}

[1] Y. Nakaki et al., Extended Abstracts ( The 35th Spring Meeting, 1988 ); The Japan Society of Applied Physics and Related Societies, P.872, ( 1988 ).

\section{ACKNOWLEDGMENTS}

The authors wish to thank Prof. T. Kunio of Keio Univ., for his advice on theory of structures.

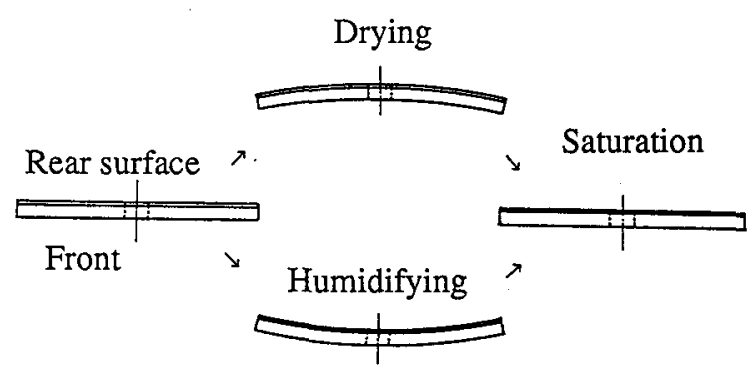

Fig. 1. Deflection in a one side recordable magneto-optical disk, induced by humidity changes.

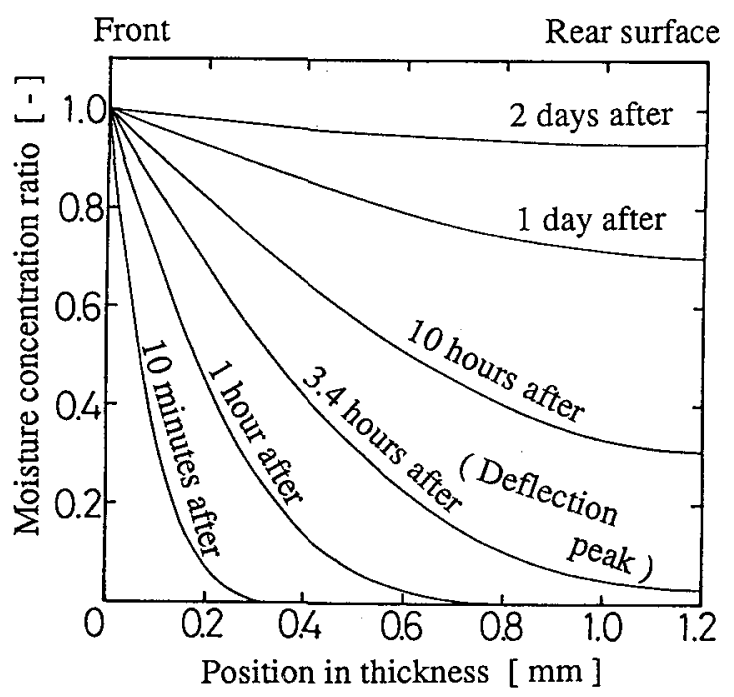

Fig. 2. Variation in moisture concentration distribution in PC substrate induced by a humidity change. (Diffusion coefficient $=10^{-7} \mathrm{~cm}^{2} / \mathrm{s}$ )

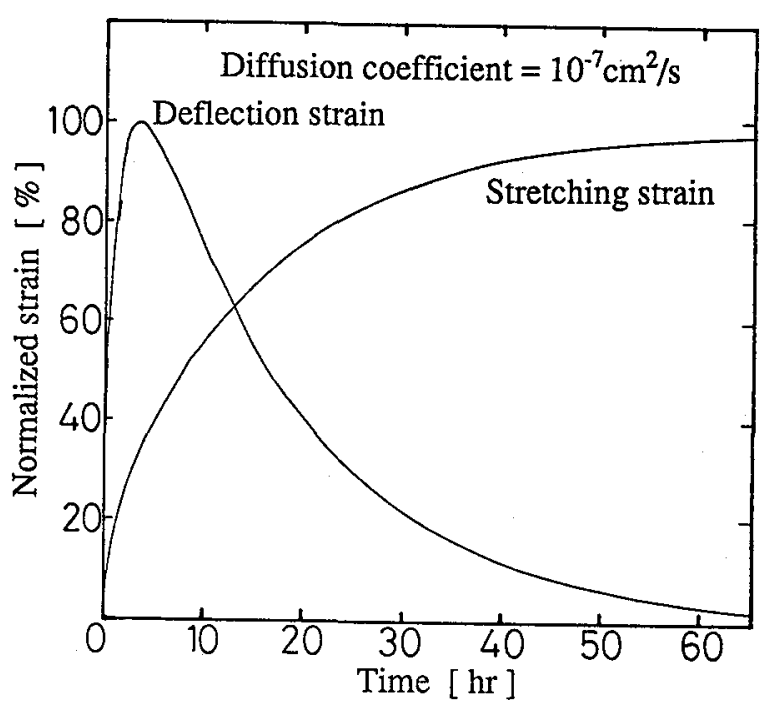

Fig. 3. Analyzed deflection and stretching strain behaviors for the disk. 


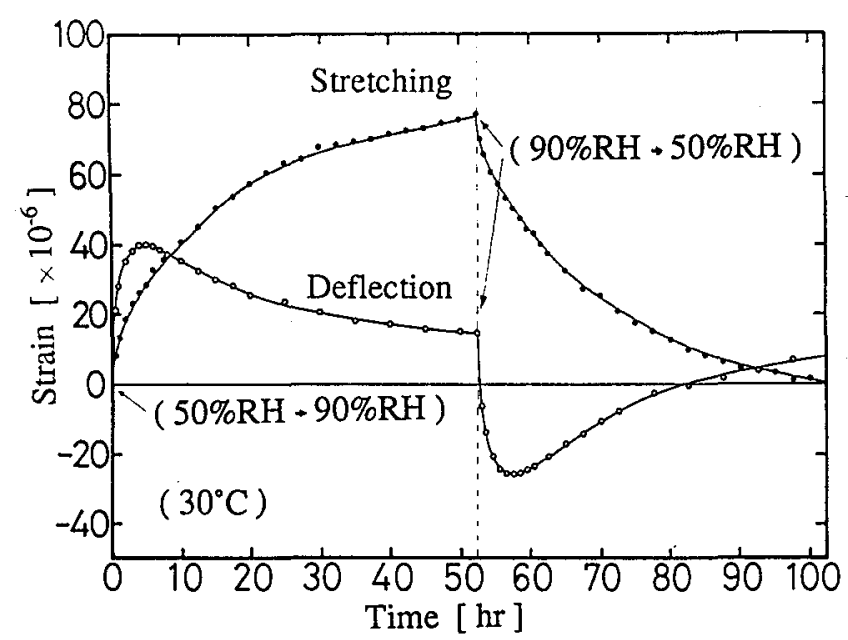

Fig. 4. Deflection and stretching strain behaviors for the disk, induced by humidity change.

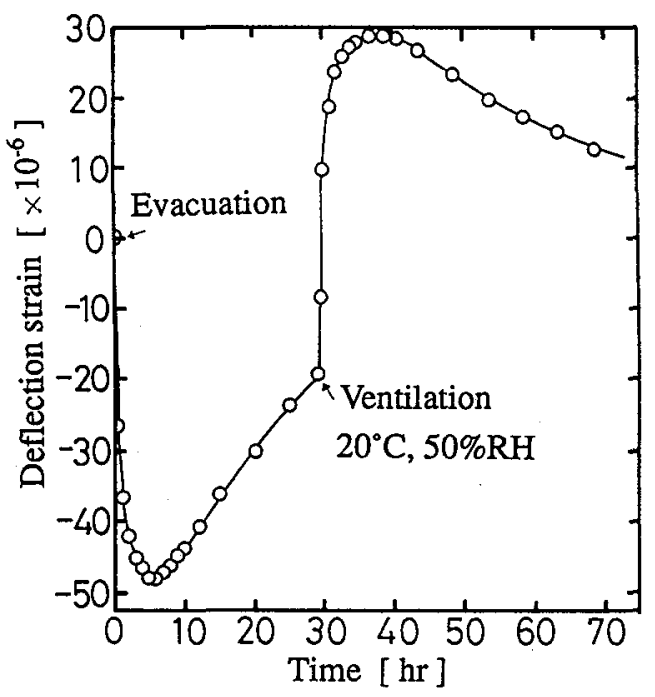

Fig. 5. Deflection strain in the disk, induced by evacuation and ventilation.

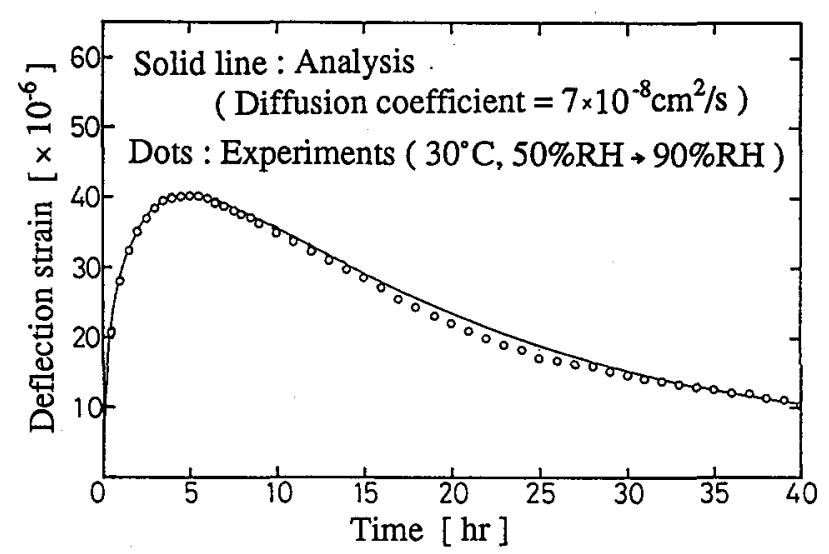

Fig. 6. Deflection behavior comparison between experiments and analysis.

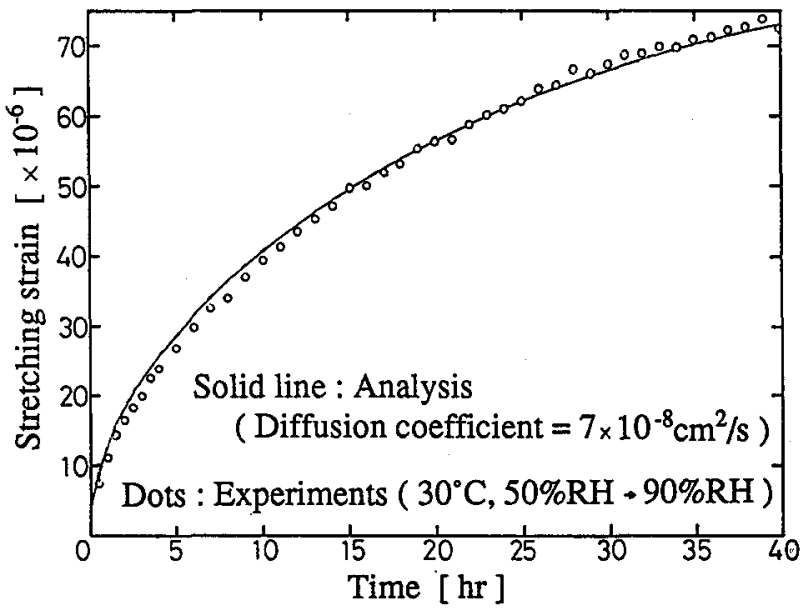

Fig. 7. Stretching behavior comparison between experiments and analysis.

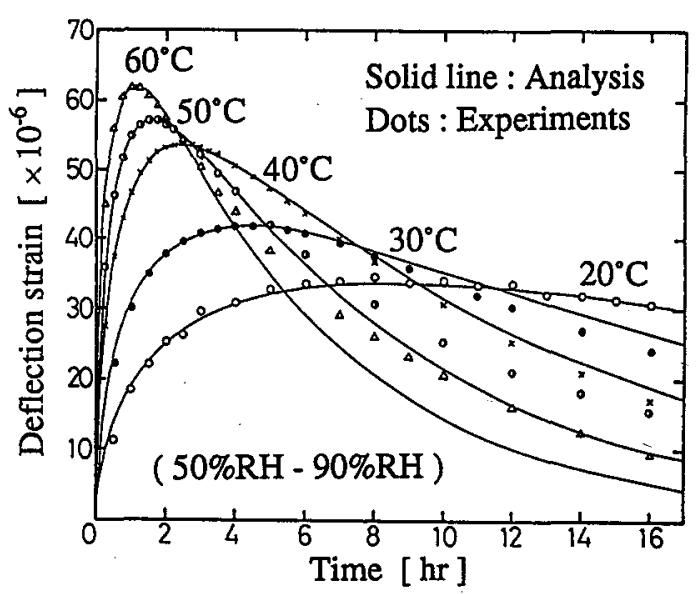

Fig. 8. Deflection strain behaviors for the disk at $20^{\circ} \mathrm{C}-60^{\circ} \mathrm{C}$ temperatures.

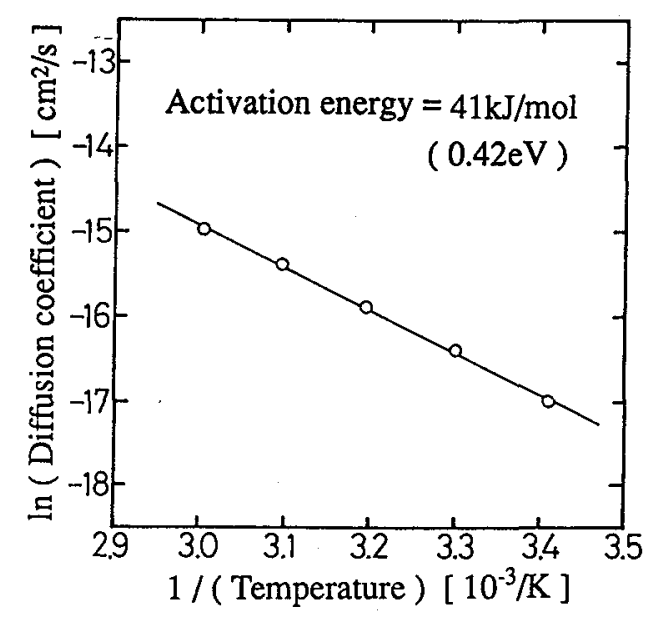

Fig. 9. Arrhenius plot for diffusion coefficients 